\title{
The FAST in screening for at-risk drinking among middle-aged women
}

\section{Tuunanen, Mervi}

2019-09-03

Tuunanen , M , Aalto , M , Levola , J \& Seppa , K 2019 , ' The FAST in screening for at-risk drinking among middle-aged women ', Journal of Substance Use , vol. 24 , no. 5 , pp. 571-574 . https://doi.org/10.1080/14659891.2019.1630679

http://hdl.handle.net/10138/319019

https://doi.org/10.1080/14659891.2019.1630679

acceptedVersion

Downloaded from Helda, University of Helsinki institutional repository.

This is an electronic reprint of the original article.

This reprint may differ from the original in pagination and typographic detail.

Please cite the original version. 
The FAST in screening for at-risk drinking among middle-aged women

Mervi Tuunanena,b*, Mauri Aalto ${ }^{\mathrm{a}, \mathrm{c}}$, Jonna Levolad and Kaija Seppäa,e

aFaculty of Medicine and Health Technology, Tampere University, Finland

bDepartment of Physiatry and Rehabilitation, South Ostrobothnia Hospital District, Finland

cDepartment of Psychiatry, South Ostrobothnia Hospital District, Finland

${ }^{d}$ Unit of Pyschiatry, Hyvinkää Area, Hospital District of Helsinki and Uusimaa, Finland

e Department of Psychiatry, Tampere University Hospital, Finland

*Corresponding author at: Department of Physiatry and Rehabilitation, South Ostrobothnia Hospital District, Huhtalantie 53, FI-60220 Seinäjoki, Finland

Tel.: +358 443227358 (mobile)

E-mail address: mervi.tuunanen@tuni.fi 


\begin{abstract}
Objective: There is a need for brief methods to screen for at-risk drinking. The FAST is a twostepped structured questionnaire. In the FAST-1, one question categorizes into three groups: lowrisk drinking, potential at-risk drinking or at-risk drinking. In the FAST-2, those with potential atrisk drinking are asked three additional questions. The aim was to study its effectiveness in screening for at-risk drinking among women and to define an optimal cut-off score.

Method: The FAST was validated against the Timeline Followback (TLFB) utilizing data from a health check of a group of 40-year old women (response rate 69.2\%; $n=907 / 1311$ ). The TLFBbased definition of at-risk drinking was consuming $\geq 140$ grams of alcohol weekly $(6.1 \%$ reported at-risk drinking).

Results and conclusions: Of all women, $54.5 \%$ could be correctly classified either as having lowrisk or at-risk drinking with the FAST-1. The optimal cut-off score was $\geq 2$ (sensitivity 0.82 , specificity 0.86 ) which is lower than has previously been reported. Only those with a FAST-1 score of one needed further evaluation with the FAST-2. A FAST-2 score of $\geq 1$ resulted in a positive screen for at-risk drinking. The FAST seems to be a valid and feasible method in screening for atrisk drinking among middle-aged women.
\end{abstract}




\section{Introduction}

At-risk drinking is defined as alcohol consumption that considerably increases the risk of negative health consequences. At-risk drinking is a common public health problem and it is often encountered in health care (Rehm et al., 2009).

Screening and brief intervention, an effective treatment for early-phase at-risk drinking, are often considered difficult by health care professionals (Hammersley, 1994; Roche \& Freeman 2004; Saunders, Aasland, Babor, de la Fuente, \& Grant, 1993). The reasons for this have been extensively studied and include professionals' negative attitudes and patients' unwillingness to reveal the correct drinking amounts, but also lack of good screening tools (Aalto, Pekuri, \& Seppä, 2001; Anderson et al., 2004; Hammersley, 1994; Kaner, Haighton, \& McAvoy, 1998; Kääriäinen, Sillanaukee, Poutanen, \& Seppä, 2001). Because clinical work is usually hectic, there is a need for brief screening methods which would be feasible for everyday work.

The Alcohol Use Disorders Identification Test (AUDIT) questionnaire has been studied widely (Berner, Kriston, Bentele, \& Härter, 2007; Reinert \& Allen 2007; Saunders et al., 1993). Its ability to screen for at-risk drinking in different clinical settings and cultures has been shown to be a good. However, it is sometimes considered too long and time-consuming for busy clinical work. This is why abbreviated AUDIT-based questionnaires have been developed (Kriston, Hölzel, Weiser, Berner, \& Härter, 2008; Meneses-Gaya et al., 2010; Reinert \& Allen 2007).

The FAST questionnaire includes four of the original ten AUDIT-questions (Table 1) (Hodgson et al., 2002). The unique feature of the FAST is that part of patients can be classified using just one question concerning the frequency of consuming six or more drinks per drinking occasion (FAST1) and only part of the patients need to respond to the remaining three questions (FAST-2) (Hodgson et al., 2002; Bradshaw el al., 2008; Gomez et al., 2005). This makes the FAST quick to administer and it is recommended for clinical use e.g. in the United Kingdom by the National Institute of Health and Clinical Excellence (Fast Screening for Alcohol Problems, 2016).

Few studies have evaluated the ability of the FAST to screen for at-risk drinking and they have mostly been done among men and validated against the AUDIT (Hodgson et al., 2003; Bradshaw el al., 2008; Gomez et al., 2005). Little is known about how the FAST performs among women.

The aim of this study was to evaluate the validity of the FAST and to define optimal cut-off scores for the FAST-1 and FAST-2 in screening for at-risk drinking among middle-aged women. 


\section{Materials and methods}

\section{Study sample}

In the city of Tampere, Finland, parts of the inhabitants are annually invited to a health check carried out in primary health care. The present study sample consisted of age group of 40-year old women attending the health check. Before attending the health check, participants were mailed a health questionnaire. It included a wide set of questions targeting different lifestyle and other issues (diet, exercise, smoking, alcohol consumption, existing diseases etc.). The questionnaire also included the complete AUDIT from which the FAST was derived.

At the health check, the participants were interviewed by nurses, who were trained to perform health screening and give counseling regarding different health habits (Aalto, Tuunanen, Sillanaukee, \& Seppä, 2006). The interview included an evaluation of the participant's alcohol consumption during the past month using the Timeline Followback (TLFB) (Sobell, L.C., Sobell, Leo, \& Cancilla, 1988).

In the TLFB interview, the participants were asked to answer in terms of Finnish standard drinks (33cl of beer, $12 \mathrm{cl}$ of wine, $4 \mathrm{cl}$ of spirits) equivalent to ca. 12 grams of absolute alcohol. Reported standard drinks were translated into grams and mean weekly alcohol consumption was calculated.

Of the 1311 invited women, 1019 attended the health check. The final sample of 907 (69.2\%) consisted of those participants, who had completed the AUDIT and for whom the results of the TLFB interview were available.

\section{Scoring of the FAST}

In this study, scoring of the FAST questionnaire was done in the way presented by Hodgson et al. (2002) (Table 1). The FAST includes two steps: FAST-1 and FAST-2. Depending on the scores of FAST-1, the participants were classified into three groups: having low risk drinking including abstinent, potential at-risk drinking or at-risk drinking. The participants who classified into the potential at-risk drinking group, were then further evaluated using the FAST-2-questions (Table 1). The final FAST scores consist of either only the FAST-1 or the sum of FAST-1 and FAST-2. 


\section{Statistical analysis}

The FAST was evaluated against the TLFB. In the TLFB, a mean $\geq 140$ grams of weekly alcohol consumption was considered at-risk drinking. This at-risk drinking limit is in accordance with the Finnish guidelines for women (Treatment of alcohol abuse. Current Care Guidelines, 2018). All possible score combinations (FAST-1 and FAST-2) were evaluated. The sensitivities, specificities, as well as positive and negative predictive values were calculated.

\section{Results}

Mean $( \pm$ SD, range) weekly alcohol consumption of the participants $(n=907)$ was 45 grams $( \pm 66,0$ 936). Of the participants, $18.1 \%$ (164/907) reported no alcohol consumption, $75.9 \%(688 / 907)$ reported consuming $>0$ but $<140$ grams and $6.1 \%$ (55/907) reported consuming $\geq 140$ grams of alcohol weekly. The mean ( \pm SD, range) AUDIT score was $3.6( \pm 3.2,0-28)$.

The best combination of sensitivity and specificity (both >0.80) was achieved with the cut-off score of $\geq 2$ (Table 2). The positive predictive values were low, but negative predictive values were equally high with all the cut-off scores. The optimal cut-off score $\geq 2$ means that only those having a score of 1 in the FAST-1 needed further evaluation by the three further FAST-2 questions. If any of the FAST-2 questions gave at least one point (FAST-2 score $\geq 1$ ), the total test was positive for atrisk drinking (Table 3).

With the most optimal cut-off score $\geq 2,54.5 \%$ of all participants (494/907) could be correctly classified either as having low-risk or at-risk drinking by the FAST-1 (Figure). Based on the FAST$1,62.6 \%(568 / 907)$ of the participants were classified as having low-risk (FAST-1 score 0) or atrisk (FAST-1 score $\geq 2$ ) drinking (Figure). Of them, 87.0\% (494/568) were correctly classified, $92.9 \%$ (459/494) belonging to the low-risk and 7.1\% (35/494) to the at-risk drinking group. In the FAST-1, 37.4\% (339/907) had a score of one and needed further evaluation using the FAST-2. Of these participants, $94.7 \%$ (321/339) were low-risk and 5.3\% (18/339) at-risk drinking according to the TLFB (Figure). 


\section{Discussion}

Of all the participants, 55\% could be correctly classified as having low-risk or at-risk drinking with a single question by the FAST-1.Based our results only those women with a score of 1 in the FAST1 need to be evaluated by using the three further FAST-2 questions.

The optimal cut-off score of the total FAST (sum of FAST-1 and FAST-2) was $\geq 2$ in the present study and was different than the cut-off score $\geq 3$ which has been recommended in previous literature (Table 2 and 3) (Hodgson et al., 2002). The previous recommendation has been that those scoring 1 or 2 in the FAST-1 are further evaluated with the FAST-2, however the present results suggest that only those with a score of 1 needed to be further evaluated (Table 3 ). These differences between the present study and previous results could be explained by the fact that the present study included only women, but Hodgson et al. (2003) reported the combined results of men and women. It seems that the cut-off scores of FAST, similarly to the AUDIT questionnaire, are different for men and women (Aalto et al., 2009).

The present results regarding the optimal cut-offs sensitivity (0.82) and specificity (0.86) were somewhat lower than in previous literature (0.89 and 0.90, respectively) (Hodgson et al., 2003). The present result, which allowed 55\% of participants to be correctly classified, based on FAST-1 is in accordance with previous results (Hodgson et al., 2002).

Other studies regarding the validity of the FAST are not directly comparable with the present results. A Spanish group studied a modified FAST-version in which the limit for binge drinking in the FAST-1 was $\geq 8$ (Gomez et al., 2005) compared to $\geq 6$ drinks, which was used in the present study and by Hodgson et al. (2003). With a cut-off score of $\geq 3$, the sensitivity was 0.80 and specificity 0.94 , but results were not reported separately for women. In an Irish study using the same FAST-questionnaire as the present study, sensitivity was 0.89 and specificity 0.94 with a cutoff score of $\geq 3$, but once again, the results were not reported separately for women (Bradshaw el al., 2008).

Because only one age group was included in the present study, these results may not be generalizable to all women. However, the very good response rate was a strength in this study. A further strength was that validation of the FAST was done against the TLFB and not against the AUDIT as in the previous studies (Bradshaw el al., 2008; Gomez et al., 2005; Hodgson et al., 2003). As far as we know, this is the first study which evaluates the validity of the FAST using the TLFB as a gold standard among women. 
Even though experienced nurses collected alcohol related data, it is still possible that participants did not report their alcohol use correctly. Underreporting is a concern especially among those having at-risk drinking. However, we estimate that possible underreporting did not affect the cut-off that was found to be optimal. In the present study, the FAST was derived from the AUDIT and effect of this on the results is unknown.

When comparing our results to the literature, it seems that the FAST questionnaire is as effective as the AUDIT in screening for at-risk drinking among women (Aalto et al., 2006, Reinert \& Allen 2007). The additional benefit of the FAST is that it is more suitable in hectic clinical work, because one question is enough for screening purposes in the majority of cases.

\section{Disclosure of interest}

The authors report no conflict of interest.

\section{References}

Aalto, M., Alho, H., Halme, J., \& Seppä, K. (2009). AUDIT and its abbreviated versions in detecting heavy and binge drinking in a general population survey. Drug Alcohol Depend, 103, 25 9.

Aalto, M., Pekuri, P., \& Seppä, K. (2001). Primary health care nurses' and physicians' attitudes, knowledge and beliefs regarding brief intervention for heavy drinkers. Addiction, 96, 305-11.

Aalto, M., Tuunanen, M., Sillanaukee, P., \& Seppä, K. (2006). Effectiveness of structured questionnaires for screening heavy drinking in middle-aged women. Alcohol Clin Exp Res, 30, 1884-8.

Anderson, P., Kaner, E., Wutzke, S., Funk, M., Heather, N., Wensing, M., Pas, L. (2004). Attitudes and managing alcohol problems in general practice: an interaction analysis based on findings from a WHO collaborative study. Alcohol Alcohol, 39, 351-6.

Berner, M. M., Kriston, L., Bentele, M., \& Härter, M. (2007). The alcohol use disorders identification test for detecting at-risk drinking: a systematic review and meta-analysis. $J$ Stud Alcohol Drugs, 68, 461-73. 
Bradshaw, P., Denny, M., \& Cassidy, E. M. (2008). Alcohol misuse in the general hospital: some hard facts. Ir J Med Sci, 177, 339-42.

Fast Screening for Alcohol Problems: Manual for the Fast Alcohol Screening Test. Retrieved June 27, 2018, available at: http://www.dldocs.stir.ac.uk/documents/fastmanual.pdf

Gomez, A., Conde, A., Santana, J. M., \& Jorrin, A. (2005). Diagnostic usefulness of brief versions of Alcohol Use Disorders Identification Test (AUDIT) for detecting hazardous drinking in primary care settings, J Stud Alcohol Drugs, 66, 305-8.

Hammersley, R. (1994) A digest of memory phenomena for addiction research. Addiction, 89, 28393.

Hodgson, R., Alwyn, T., John, B., Thom, B., \& Smith, A. (2002). The FAST Alcohol Screening Test. Alcohol Alcohol, 37, 61-6.

Hodgson, R. J., John, B., Abbasi, T., Hodgson, R. C., Waller, S., Thom, B., \& Newcombe, R. G. (2003). FAST screening for alcohol misuse. Addict Behav 2003, 28, 1453-63.

Kaarne, T., Aalto, M., Kuokkanen, M., \& Seppä, K. (2010) AUDIT-C, AUDIT-3 and AUDIT-QF in screening risky drinking among Finnish occupational health care patients. Drug Alcohol Rev, 5, 563-7.

Kaner, E. F., Haighton, C. A., \& McAvoy, B. R. (1998). 'So Much post, so busy with practice-so, no time!': a telephone survey of general practioners' reasons for not participating in postal questionnaire surveys. Br J Gen Pract, 48, 1067-9.

Kriston, L., Hölzel, L.,Weiser, A. K., Berner, M. M., \& Härter, M. (2008). Meta-analysis: Are 3 questions enough to detect unhealthy alcohol use? Ann Intern Med, 149, 879-88.

Kääriäinen, J., Sillanaukee, P., Poutanen, P., \& Seppä K. (2001) Opinions on alcohol-related issues among professionals in primary, occupational, and specialized health care. Alcohol Alcohol, 36, 141-6.

Meneses-Gaya, C., Zuardi, A.W., Loureiro, S.R., Hallak, J. E., Trzesniak, C., de Azevedo Marques, J. M., Crippa, J. A. (2010). Is the full version of the AUDIT really necessary? Study of the validity and internal construct of its abbreviated versions. Alcohol Clin Exp Res, 34, 1417-24. 
Rehm, J., Mathers, C., Popova, S., Thavorncharoensap, M., Teerawattananon, Y., Patra, J. (2009). Global burden of disease and injury and economic cost attributable to alcohol use and alcohol-use disorders. Lancet, 373, 2223-33.

Reinert, D. F., \& Allen, J. P. (2007). The Alcohol Use Disorders Identification Test: an update of research findings. Alcohol Clin Exp Res, 31, 185-99.

Roche, A. M., \& Freeman, T. (2004). Brief interventions: good in theory but weak in practice. Drug Alcohol Rev, 23, 11-8.

Saunders, J. B., Aasland, O. G., Babor, T. F., de la Fuente, J. R., \& Grant, M. (1993). Development of the Alcohol Use Disorders Identification Test (AUDIT): WHO Collaborative Project on Early Detection of Persons with Harmful Alcohol Consumption - II. Addiction, 88, 791-804.

Sobell, L.C., Sobell, M. B., Leo, G. I., \& Cancilla, A. (1988). Reliability of a timeline method: assessing normal drinkers` reports of recent drinking and a comparative evaluation across several populations. Br J Addict, 83, 393-402.

Tuunanen, M., Aalto, M., Seppä, K. (2007). Binge drinking and its detection among middle-aged men using AUDIT, AUDIT-C and AUDIT-3. Drug Alcohol Rev, 26, 295-9.

Working group appointed by the Finnish Medical Society Duodecim and the Finnish Society of Addiction Medicine. Treatment of alcohol abuse. Current Care Guidelines. (2018). Available at: http://www.kaypahoito.fi/web/kh/suositukset/suositus?id=hoi50028 
Table 1. The FAST questionnaire and its scoring.

FAST-1

1. How often do you have 6 or more drinks on one occasion?

Never Less than monthly Monthly Weekly Daily or almost daily (scoring 0, 1, 2, 3, 4)

\section{FAST-2}

2. How often during the last year have you been unable to remember what happened the night before because you had been drinking?

Never Less than monthly Monthly Weekly Daily or almost daily (scoring 0, 1, 2, 3, 4)

3. How often during the last year have you failed to do what was normally expected of you because of drinking?

Never Less than monthly Monthly Weekly Daily or almost daily (scoring 0, 1, 2, 3, 4)

4. In the last year has a relative or friend, or a doctor or other health worker been concerned about your drinking or suggested you cut down?

No Yes, on one occasion Yes, on more than one occasion (scoring 0, 2, 4) 
Table 2. The sensitivities, specificities, positive and negative predictive values of the FAST questionnaire using different cut-off scores. Cut-offs refer to FAST-1 and FAST-2 sum score.

\begin{tabular}{lllll}
\hline Cut-off & Sensitivity & Specificity & $\begin{array}{l}\text { Positive } \\
\text { predictive } \\
\text { value }\end{array}$ & $\begin{array}{l}\text { Negative } \\
\text { predictive } \\
\text { value }\end{array}$ \\
\hline$\geq 1$ & 0.96 & 0.53 & 0.12 & 0.99 \\
$\geq 2$ & 0.82 & 0.86 & 0.27 & 0.99 \\
$\geq 3 *$ & 0.60 & 0.94 & 0.38 & 0.97
\end{tabular}

*Best cut-off by Hodgson et al. 2003 
Table 3. The cut-offs of FAST in the previous and the present study.

Hodgson et al., $2003 \quad$ Present study

FAST-1 score

Low-risk drinking

0

0

Potential at-risk drinking,

further evaluated by the FAST-2

$1-2$

1

At-risk drinking

3-4

$2-4$

FAST-2 score

Low-risk drinking

$0-1$

0

At-risk drinking

$\geq 1-2$

$\geq 1$

FAST-1 and FAST-2 sum score

Low-risk drinking

$<3$

$<2$

At-risk drinking

$\geq 3 \quad \geq 2$

Maximum score

14

13 
Figure. The validity of FAST questionnaire (cut off score $\geq 2$ ) in screening at-risk drinking.

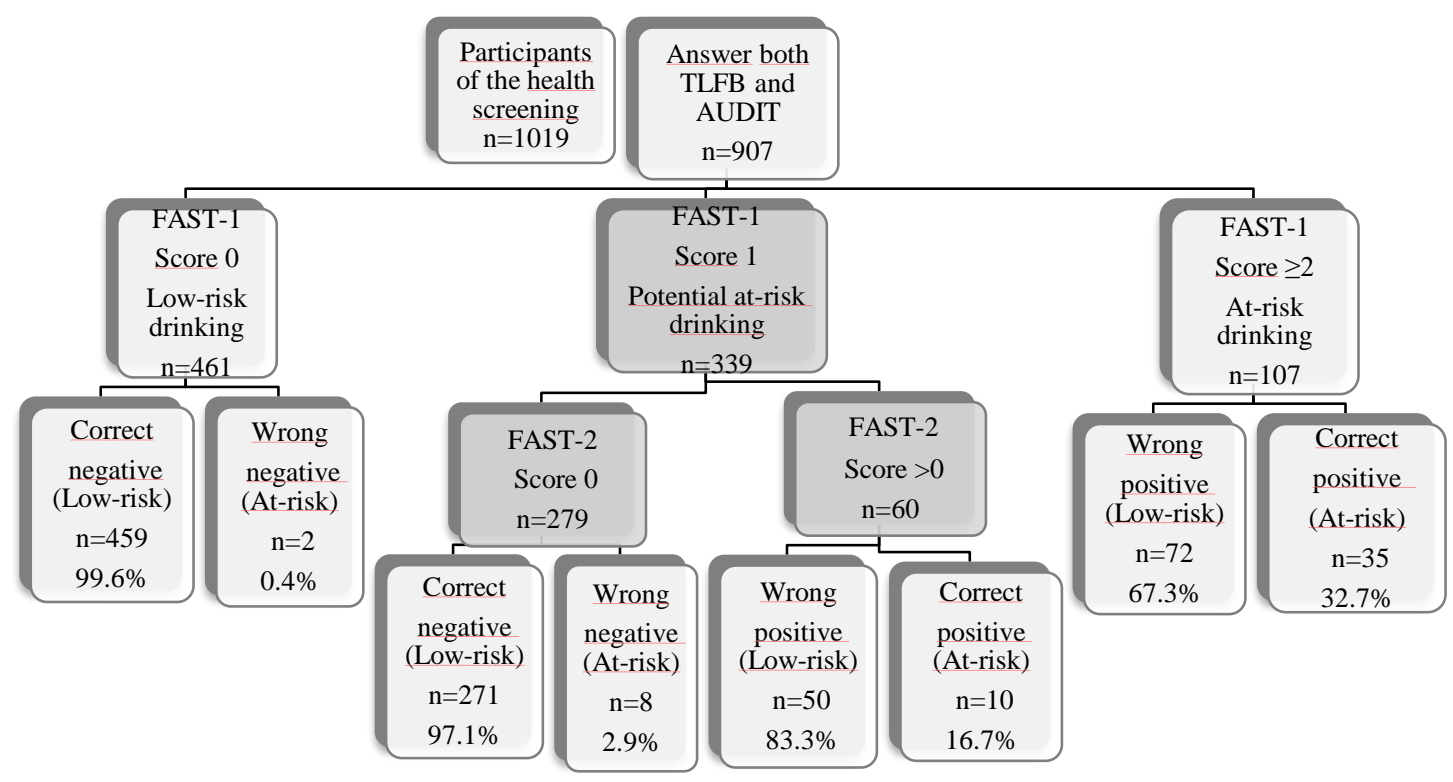

